\title{
On rate-dependent mechanical model for adaptive magnetorheological elastomer base isolator
}

\author{
Yancheng $\mathrm{Li}^{*}$ and Jianchun $\mathrm{Li}$ \\ Centre for Built Infrastructure Research, School of Civil and Environmental Engineering, Faculty of Engineering and \\ Information Technology, University of Technology Sydney, NSW 2007, Australia \\ Email: Yancheng.li@uts.edu.au
}

\begin{abstract}
:
This paper presents research on the phenomenological model of an adaptive base isolator. The adaptive base isolator is made of field-dependent magnetorheological elastomer (MRE) which can alter its physical property under application of magnetic field. Experimental testing demonstrated that the developed MRE base isolator possesses an amazing ability to vary its stiffness under applied magnetic field. However, several challenges have been encountered when it comes modeling such novel device. For example, under a large deformation, the MRE base isolator exhibits a clear strain stiffening effect and this behavior escalates with the increasing of applied current. In addition, the MRE base isolator has also shown typical rate-dependent behavior. Following a review on mechanical models for viscos-elastic rubber devices, a novel rate-dependent model is proposed in this paper to capture the behavior of the new MRE base isolator. To develop a generalized model, the proposed model was evaluated using its performance under random displacement input and a seismic input. It shows that the proposed rate-dependent model can successfully describe the complex behavior of the device.
\end{abstract}

Keywords: base isolator; magnetorheological elastomer; mechanical model; variable stiffness;

\section{Introduction:}

As an important technique of seismic protection, base isolation has been widely adopted in protecting civil structures against earthquakes [1-2]. The fundamental principle for a base isolation system for mitigating earthquake hazards is to decouple the undesirable motion caused by earthquake and thus to avoid the transition of these destructive effect into main structure [3-6]. The traditional passive base isolation systems have been challenged for lacking of adaptability and effectiveness for different earthquakes. To effectively protect the structure, hybrid base isolation systems have been proposed for overcoming the challenges, including compensating traditional base isolation systems with passive, semi-active [7] and active damping components. However, additional damping only suppresses seismic responses through dissipating seismic energy [8-9]. The most important measure, i.e. fundamental frequency of base-isolated structure, does not change. Thus, this technology cannot decouple the motions between the structures and the ground. An ideal semi-active base isolation system should be able to alter its stiffness adaptively therefore it can deflect energy from ground motion in real-time. To accomplish this, a base isolation system with adaptive/variable stiffness is required. There have been efforts from researchers in pursuing variable stiffness mechanism in base isolation system. For example, Nagarajaiah and Sahasrabudhe [10] proposed a semi-active independently variable stiffness mechanism to be used in seismic response control of smart sliding isolated buildings. In general, research and development on variable stiffness systems is limited but can be found in [11-14].

Magnetorheological elastomer can be used for this purpose due to its unique properties, i.e. adjustable stiffness controlled by applied magnetic field [15-16]. MRE material composes magnetic iron particle, silicon oil and rubber matrix [17]. Three ingredients are mixed and cured into solid 
rubber compound [18]. With external magnetic field, the magnetic force between the magnetic particles causes the property changes of the rubber material, i.e. shear modulus and damping. Once removed the magnetic field, the material acts as normal rubber/elastomer material [19-23]. This realtime controllable rubber material offers potential to develop various kinds of vibration isolation devices, i.e. adaptive base isolator. The adaptive base isolator utilizes field-dependent property of magnetorheological elastomer (MRE) in order to alter its stiffness in real-time. In the innovative design of the adaptive MRE base isolator proposed by Li et al [24-25], traditional laminated multilayers structure of seismic isolator design has been adopted in order produce the needed axial loadcarry capacity for the novel adaptive isolator. The experimental testing demonstrated that the new adaptive base isolator can create significant increase on lateral stiffness for more than 16 times [25]. With such feature, a new intelligent base isolation system with real-time decoupling ability can be achieved by replacing traditional passive base isolators with the new ones.

In order to take advantage of the novel adaptive base isolator to develop a truly intelligent base isolation system that can overcome shortcoming of the traditional base isolation systems and optimally adapt to any unknown types of earthquakes, establishing an accurate and reliable model of the novel adaptive base isolator is crucial. A high fidelity model will enable a systematic investigation of the control design and evaluation of such adaptive base isolation system through simulation and therefore lead to an efficient experimental realization of the system. In this paper, experimental characterization testing for the device is briefly described and based on the experimental results the unique behavior of the MRE base isolator is discussed in detail. A new ratedependent mechanical model is proposed to capture the behavior of the proposed MRE base isolator including its strain-stiffening and field-sensitive characteristics. With incorporating a new strainstiffening element, the proposed mechanical model is able to accurately capture the nonlinear behavior of MRE base isolator.

\section{Experimental setup and testing results}

\subsection{MRE base isolator and experimental setup}

Experimental testing on MRE base isolator (figure 1) was conducted utilizing MTS shake table, as shown in figure 2. MRE base isolator was installed on top of the shake table which produces harmonic loading to the device. Power supply was used to provide fixed currents to MRE base isolator and load cell was used to capture the shear force produced. The MRE base isolator was examined in dynamic testing by applying different sinusoidal loadings with combination of loading amplitudes, i.e. $2.0 \mathrm{~mm}, 4.0 \mathrm{~mm}$ and $8.0 \mathrm{~mm}$, and loading frequencies, i.e. $0.1 \mathrm{~Hz}, 1.0 \mathrm{~Hz}, 2.0 \mathrm{~Hz}$ and $4.0 \mathrm{~Hz}$. For single sinusoidal loading, four different currents, i.e. $0.0 \mathrm{~A}, 1.0 \mathrm{~A}, 2.0 \mathrm{~A}$ and $3.0 \mathrm{~A}$, were applied. Detailed experimental setting can be found in [25].

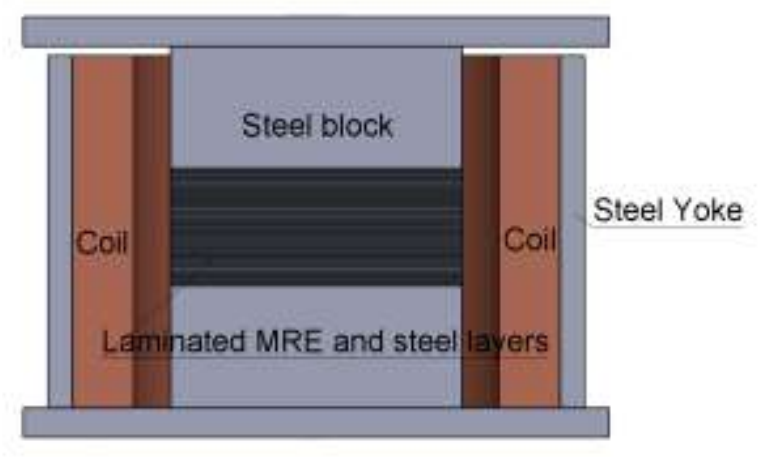

Figure 1. Sectional view of MRE base isolator (Solidworks) 


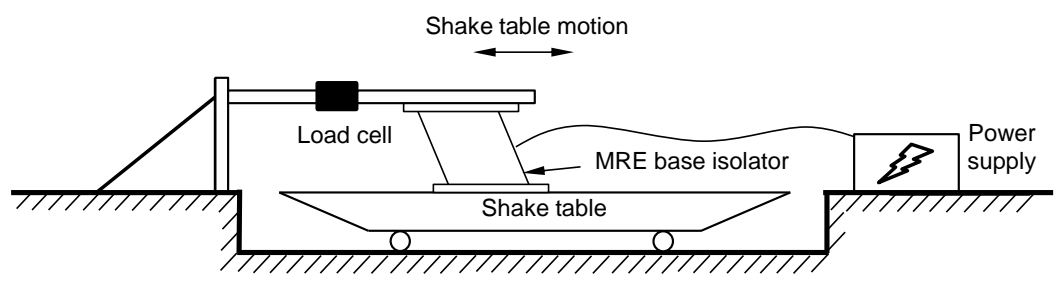

Figure 2. Sketch map of the experimental set-up

Figures 3 and 4 present the force-displacement and force-velocity relationships of the MRE base isolator at 12 sets of experimental data at loading frequency of $1.0 \mathrm{~Hz}$, i.e. $\Delta=2 \mathrm{~mm}, 4 \mathrm{~mm}$ and $8 \mathrm{~mm}$, and $\mathrm{I}=0.0 \mathrm{~A}, 1.0 \mathrm{~A}, 2.0 \mathrm{~A}$ and $3.0 \mathrm{~A}$, respectively. The figures show significant $\mathrm{MR}$ effect from $\mathrm{I}=0.0 \mathrm{~A}$ to I=3.0A. Detailed experimental results can be found in [25].
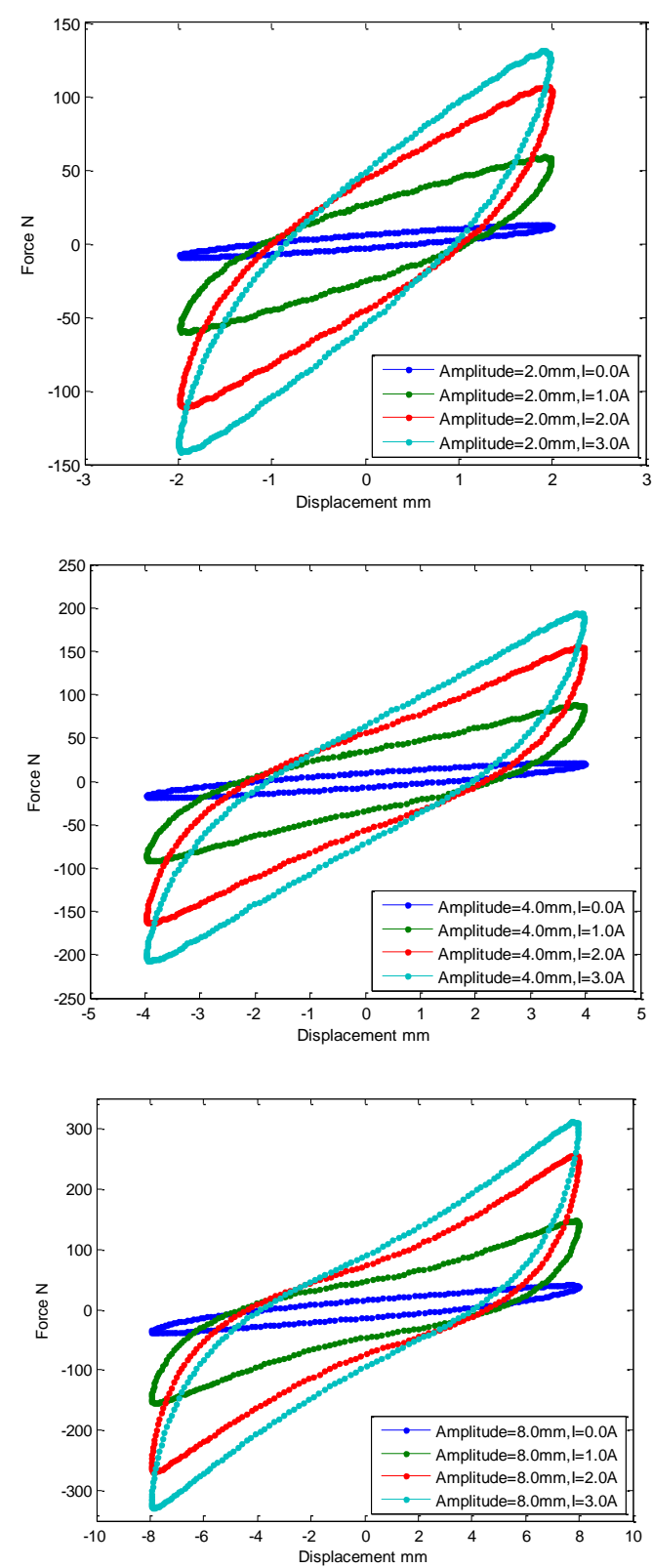

Figure 3. Force-displacement responses under different displacement $(\mathrm{f}=1.0 \mathrm{~Hz})$
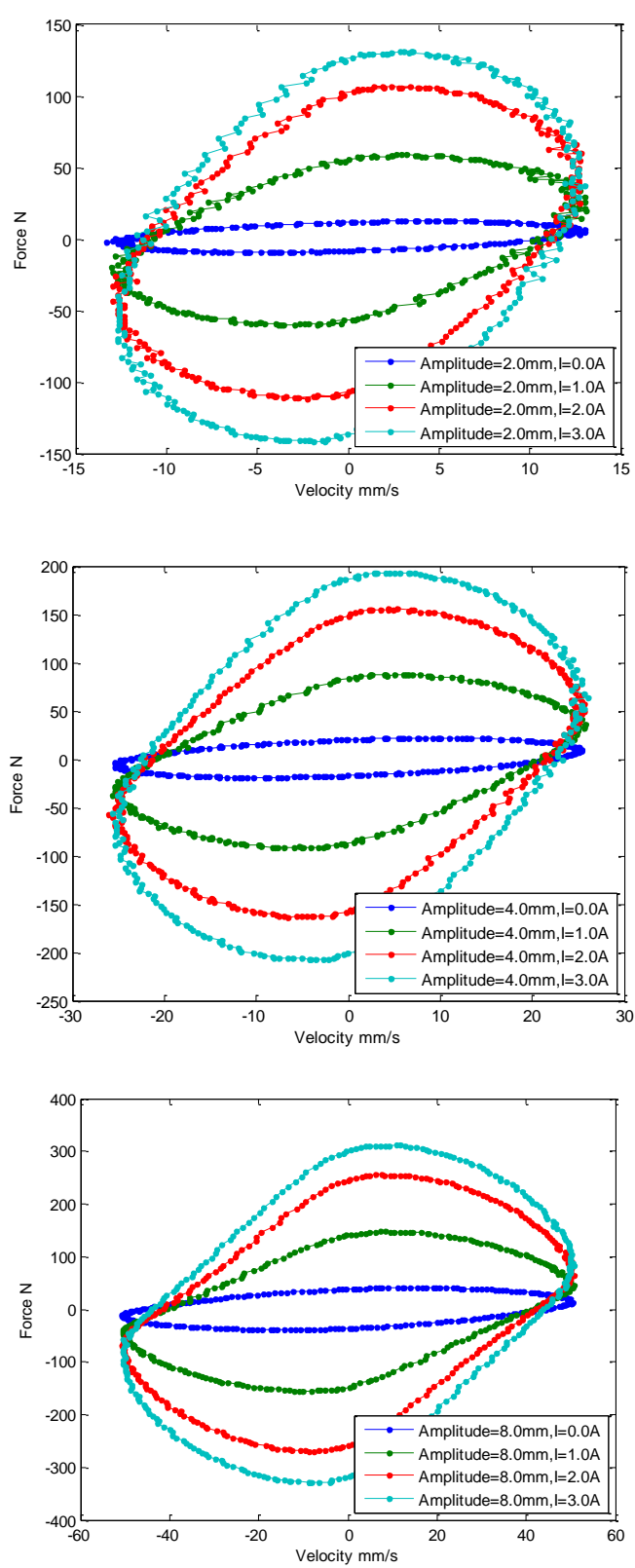

Figure 4. Force-velocity responses under different displacement $(\mathrm{f}=1.0 \mathrm{~Hz})$

\subsection{Characteristics of the MRE base isolator}


In the experimental evaluation of base isolator, stiffness and damping properties are always used to describe its characteristics [26-27]. Effective stiffness of a base isolator, as shown in figure 5, is defined by the force-displacement loops from the experimental results of cyclic testing. In [26], the effective stiffness is defined based on half of the hysteresis loop (shown in figure 5) as:

$$
K_{e f f}=\frac{F_{X^{+}}}{X^{+}}
$$

Where, $F_{X^{+}}$is the force at the maximum positive displacement $\left(\mathrm{X}^{+}\right)$. However, in the experimental testing, the hysteresis loop is not always perfectly centered with the origin. Therefore, a generalized method [27] is used to evaluate its effective stiffness from experimental data, as:

$$
K_{e f f}=\frac{F_{X^{+}}-F_{X^{-}}}{X^{+}-X^{-}}
$$

Where, $F_{X^{-}}$is the force at the minimum negative displacement $\left(\mathrm{X}^{-}\right)$. Therefore, the force bias/offset can be eliminated to improve the accuracy.

Besides the effective stiffness, dynamic stiffness is also used for the evaluation of the rubber isolator [28]. The dynamic stiffness $K_{d y n}$ is defined as:

$$
K_{d y n}=\frac{F^{+}}{X^{+}}
$$

Here, $F^{+}$is the maximum force response.

The definitions of effective stiffness and dynamic stiffness reflect the global and local stiffness of the hysteresis loop of the rubber bearing. With the assumption of adequate damping in the device, they can be used in modeling of the hysteresis behavior via skeleton curve defined by previous equations, such as the bilinear model [29] and [30].

The equivalent damping, $c_{e q}$, calculated from a force-displacement loop can be written as

$$
c_{e q}=\frac{E D C}{\pi \omega X^{2}}
$$

The damping ratio of the device is

$$
\zeta=\frac{E D C}{2 \pi K_{e f f} X^{2}}
$$

Where, $E D C$ is the energy dissipation in one cycle, $\omega$ and $X$ are the angular frequency and amplitude of the excitation, respectively.
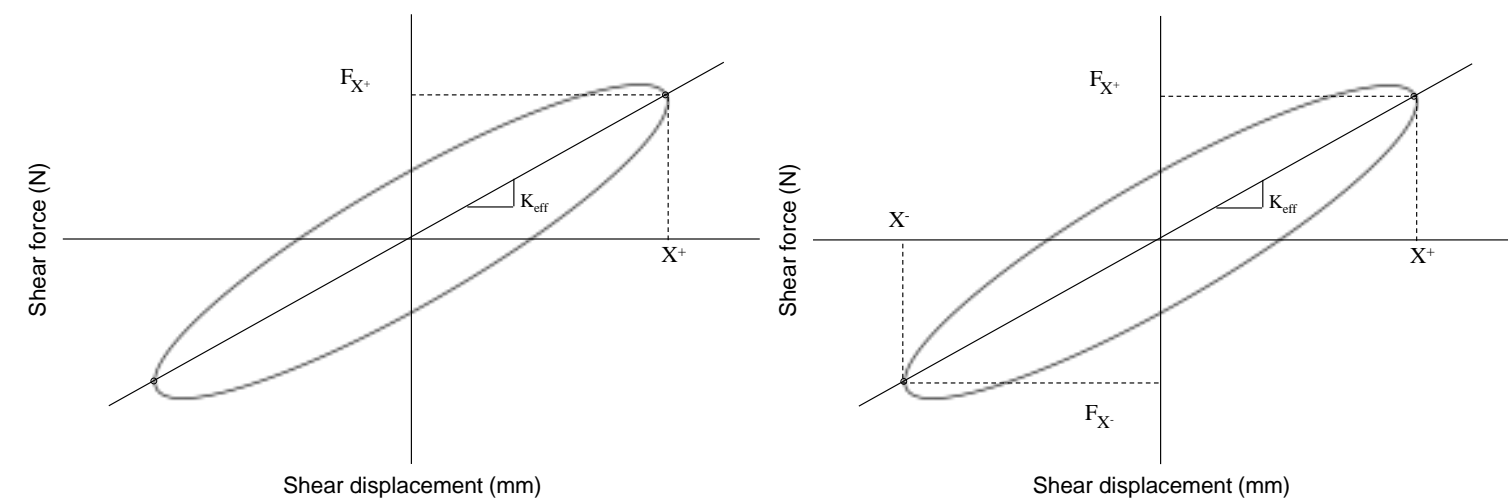

Figure 5 Definitions of effective stiffness: 1) from AASHTO [26]; 2) general method [27]

Based on the equations above, the effective stiffness and damping ratio of MRE base isolator at loading frequency of $1.0 \mathrm{~Hz}$ are listed in Table 1. 
Table 1. Effective stiffness and damping ratio (loading frequency $=1.0 \mathrm{~Hz}$ )

\begin{tabular}{cllllllll}
\hline \multirow{2}{*}{$\begin{array}{c}\text { Deformation } \\
\mathrm{mm}\end{array}$} & \multicolumn{4}{c}{ Effective stiffness $\left[\mathrm{kN} \mathrm{m}^{-1}\right]$} & \multicolumn{5}{c}{ Damping ratio } \\
\cline { 2 - 9 }$y$ & $0.0 \mathrm{~A}$ & $1.0 \mathrm{~A}$ & $2.0 \mathrm{~A}$ & $3.0 \mathrm{~A}$ & $0.0 \mathrm{~A}$ & $1.0 \mathrm{~A}$ & $2.0 \mathrm{~A}$ & $3.0 \mathrm{~A}$ \\
\hline $2 \mathrm{~mm}$ & 4.96 & 27.13 & 52.22 & 66.13 & 0.220 & 0.240 & 0.218 & 0.201 \\
$4 \mathrm{~mm}$ & 4.69 & 20.72 & 38.15 & 48.74 & 0.223 & 0.228 & 0.206 & 0.189 \\
$8 \mathrm{~mm}$ & 4.62 & 17.72 & 31.44 & 39.31 & 0.205 & 0.204 & 0.188 & 0.179 \\
\hline
\end{tabular}

\subsection{Strain-stiffening, rate-dependency and stiffness degradation}

MRE base isolator exhibits three unique characteristics, i.e. strain-stiffening, rate-dependency and stiffness degradation. Experiment reveals that MRE base isolator exhibits increasing stiffness in single force-displacement loop at large shear deformations, i.e. strain-stiffening, as shown in Figure 3. This behavior becomes more significant with magnetic field applied. Rate-dependency refers to the maximum shear force measured is dependent on the shear rate the isolator experiences, as shown in Figure 6. As observed, the rate-dependency is more obvious in zero magnetic field cases, i.e. no current is applied. Figure 7 presents the hysteresis loops of MRE base isolator and the calculated stiffness when same current and loading frequency are applied. With increasing amplitude in sinusoidal loadings, the effective stiffness gradually decreases. Detailed discussions on three phenomena can be found in [25].
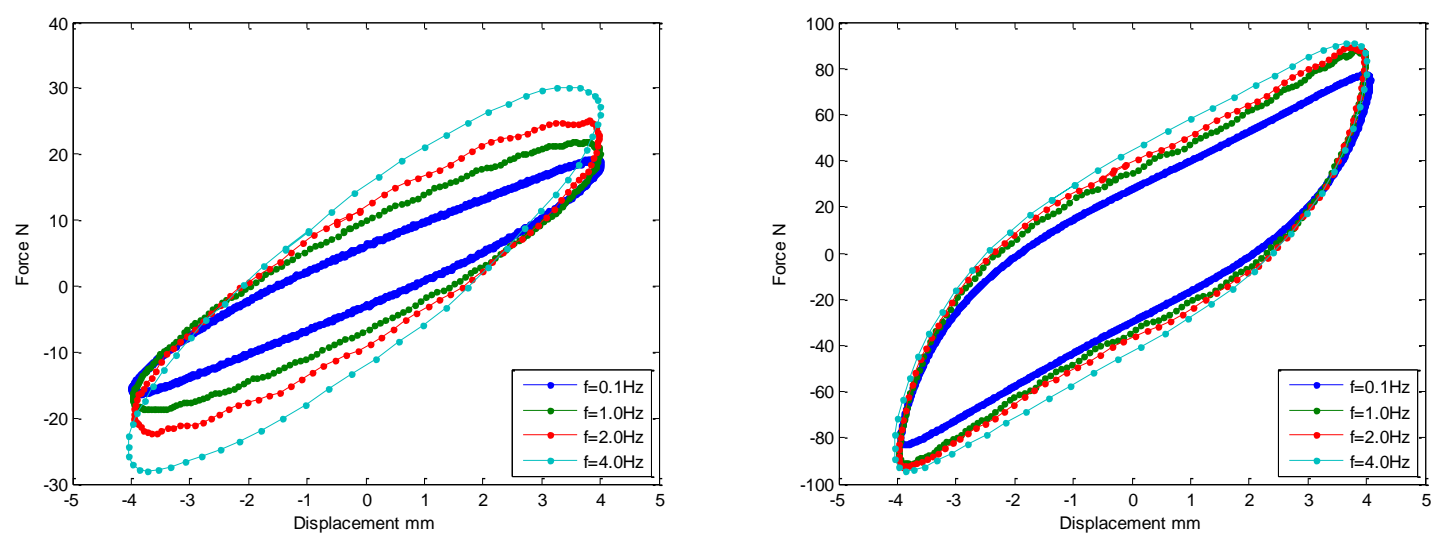

Figure 6 Rate-dependent behavior of MRE base isolator at $4.0 \mathrm{~mm}$ amplitude: (1) I=0.0A; (2) I=1.0A;

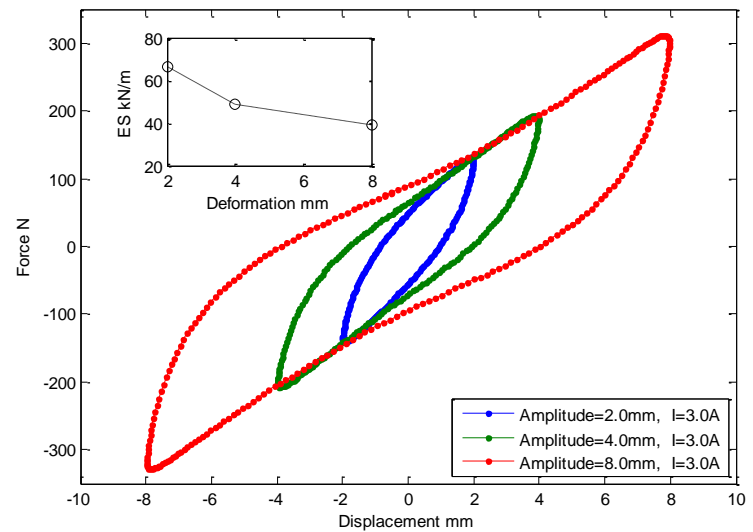

Figure 7. Stiffness degradation of the MRE base isolator $(\mathrm{f}=1.0 \mathrm{~Hz})$ 
Aforementioned behaviors form distinct features of MRE base isolator that should be considered for modeling its hysteresis. Therefore, an effective model should include elements that describe these features. Normally, rate-dependent behavior can be portrayed through establishing viscous damping element, while modeling of strain-stiffening and stiffness degradation behaviors can only be resolved by introducing new element in the mechanical model.

\section{Rate-dependent mechanical models for base isolators \\ 3.1. Standard Solid model with a strain-stiffening element}

Typical viscoelastic models and their improved versions, such as Kevin-Voigt model, standard solid model and four-parameter model, have been proposed to describe the behavior of MR elastomer and devices [20]. In Kevin model, the viscoelasticity of the rubber is defined as the combination of a linear spring and a viscos dashpot element. The linear spring represents elastic behavior while the dashpot element represents viscos behavior of the rubber material. To improve the Kevin model under sudden loading, three-parameter solid model is proposed by adding a second linear spring element in series with the Kevin model. However, these models can only portray ellipse hysteresis loop and are incapable to capture the unique strain-stiffening behavior of the MRE base isolator in this research.

Strain-stiffening refers to the phenomenon that local stiffness of the hysteresis loop increases with the shear strain. This behavior is often reported to be associated with high-damping rubber [31-33] and high-damping rubber bearings/isolators [34-48]. A number of mathematical models, including rheological models (for rubber material) [38] and constitutive/mechanical models (for rubber devices) [32-48], have been proposed to characterize the strain stiffening behavior (in some literatures, it is called strain hardening [33, 36-37]). One of the common techniques in modeling the strain stiffening behavior is to introduce nonlinear power function spring or element. Kikuchi and Aiken [34] proposed a skeleton element to characterize the hysteresis loop of rubber bearing in stiffening range. Another example in using skeleton curve for characterizing strain-stiffening is Tsai et al[35]. Iizuka [29] extended the Koh-Kelly model [48] by introducing finite element deformation and a nonlinear spring element (power function). This model can reasonably capture the mechanical behavior of the rubber bearing. Abe et al [38] extended the Ozdemir model [49] by introducing several new spring elements, including hardening spring. This hardening element also adopts power function. By identifying the initial yielding force and another two model parameters, the hardening behavior can be reproduced. However, this model involves 13 parameters which bring great computation challenge. Other examples in using power function rules in modeling of rubber bearing are [33] [39].

In this paper, a nonlinear spring is introduced based on power function which creates a smooth transition from viscoelasticity to strain stiffening behavior. The generalized form of the power law function is given as:

$$
f(x)=\alpha x^{\frac{n}{m}}
$$

Where, $\frac{n}{m}$ is the power law exponent and $\alpha$ is the scale factor parameter, $\mathrm{m}$ and $\mathrm{n}$ are positive integers. To define the hysteresis loop of the MRE isolator, one needs to satisfy the conditions as: (i) if $x>0$, then $f(x)>0$; (ii) if $x<0$, then $f(x)<0$ and (iii) the power law exponent $\frac{n}{m}>1$, thus $n>m$. Here, we define a nonlinear spring element $\boldsymbol{F}_{\text {st }}$ as:

$$
F_{s t}=\left\{\begin{array}{cl}
\alpha x^{\frac{n}{m}} & \text { if } m \text { and } n \text { are odd integers satisfying } n>m \\
\alpha|x| x^{\frac{n-m}{m}} & \text { if } m \text { is an odd integer and } n \text { is an even integer satisfying } n>m
\end{array}\right.
$$


If $\mathrm{m}=\mathrm{n}$ in equation 7 , it becomes a linear spring element. Figure 8 presents three spring elements from equation 7. In the following analysis, a power law function element $F_{s t}=\alpha x^{3}$ is introduced for all cases.
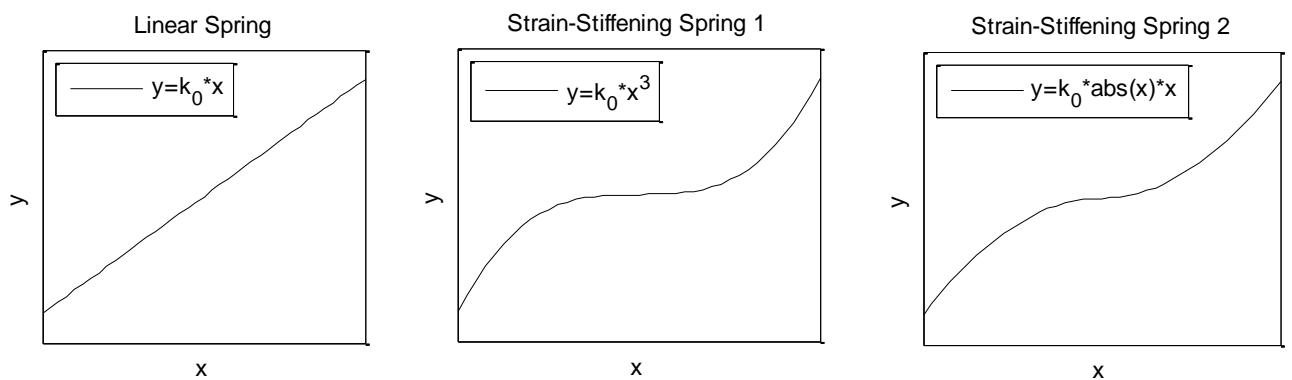

Figure 8 Spring and strain stiffening elements

Introducing the power law function element into the three parameter solid model, the new model is shown in figure 9. The mathematical expression of this model is given by:

$$
\begin{gathered}
F=k_{1} y+\alpha x^{3} \\
k_{1} y=k_{0}(x-y)+c_{0}(\dot{x}-\dot{y})
\end{gathered}
$$

Rearranging equation 9 obtains

$$
\dot{y}=-\frac{\left(k_{0}+k_{1}\right)}{c_{0}} y+\dot{x}+\frac{k_{0}}{c_{0}} x
$$

Where, $\alpha$ is the parameter for the power law element. This model contains 4 parameters, $c_{0}, k_{0}, k_{1}$ and $\alpha$, to be identified.

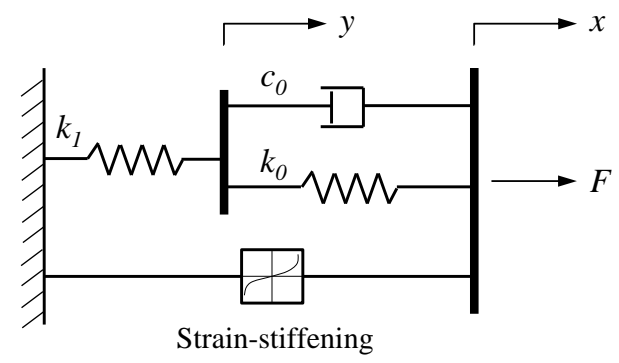

Figure 9 Mechanical model containing a strain-stiffening element
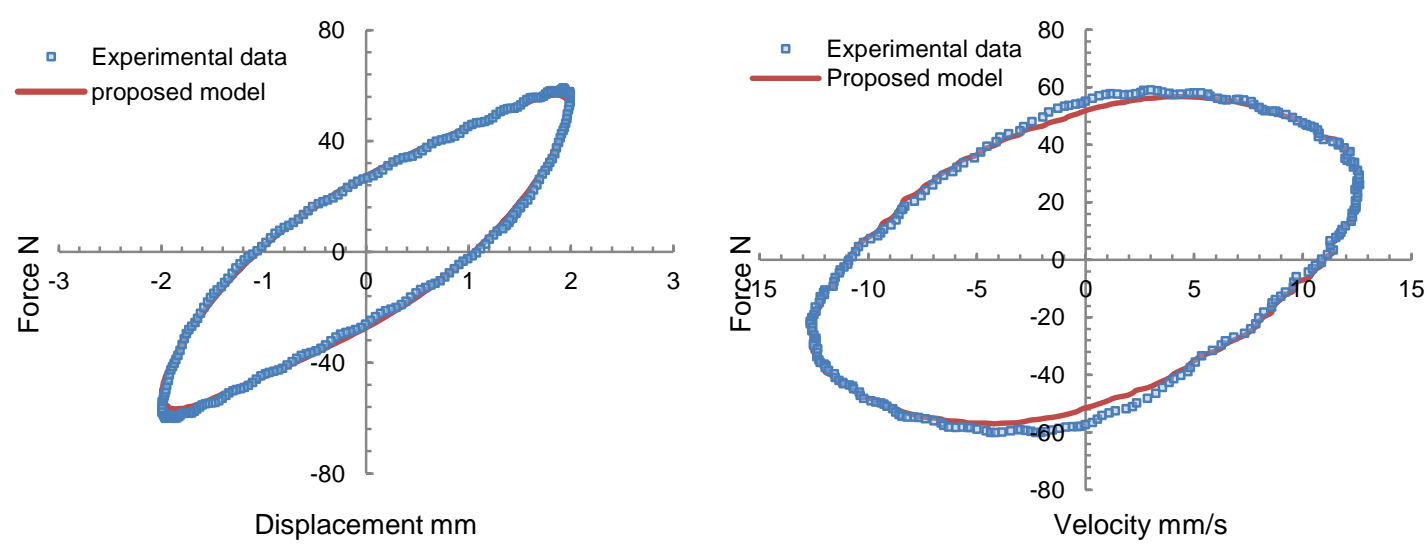

a) Small displacement 

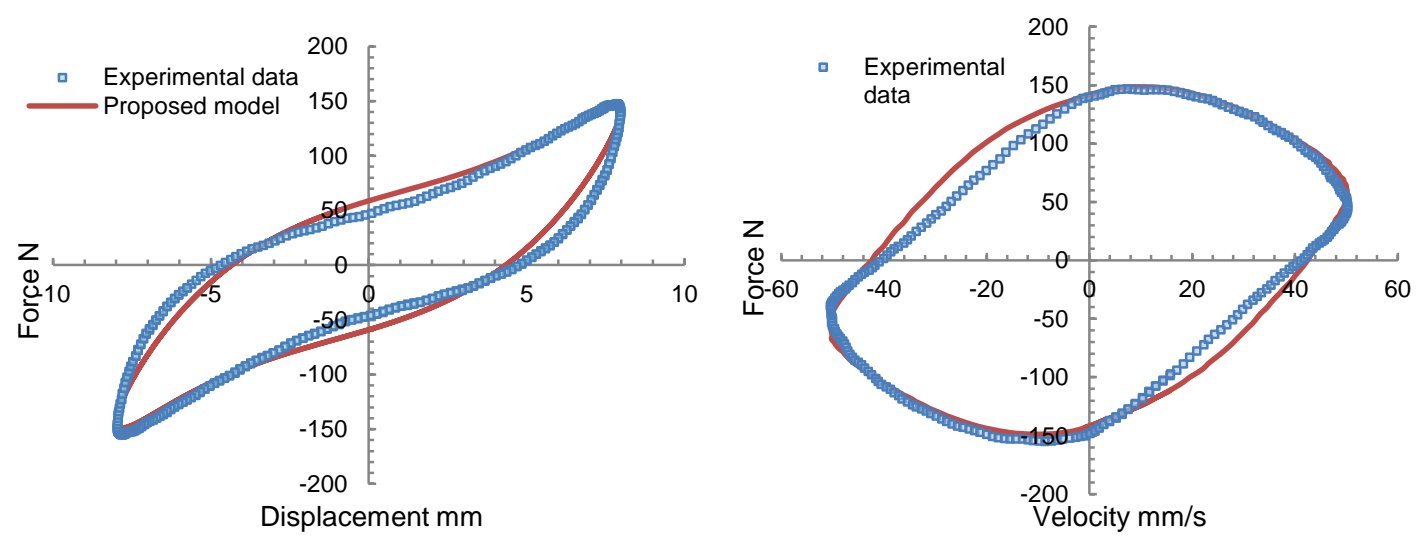

b) Large displacement

Figure 10. Comparison between the experimental data and model prediction for three parameter solid model with strainstiffening element

The model proposed in equations 11 and 12 was fit to the experimental data obtained at a frequency of $1.0 \mathrm{~Hz}$ and current of $1.0 \mathrm{~A}$. Figure 10 is the comparison between the experimental response and the proposed model for both small displacement and large displacement. Adding the strain stiffening element into the three parameter solid model greatly improves the model accuracy. The new model is able to predict the force-displacement and force-velocity responses at small displacement. However, for large displacement cases, this model is less efficiency. It cannot well characterize the forcedisplacement response when the displacement of the isolator is near zero. Accordingly, discrepancy occurs in the predicted force-velocity response. The reason behind this is due to the fact that the strain-stiffening element has a fixed turning point $(x=1)$ while the MRE base isolator possesses various critical yielding displacements for different lateral displacements.

\subsection{New strain-stiffening model}

To better predict nonlinear viscos-elastic behavior of MRE base isolator, a modified strain-stiffening model is proposed in Figure 11. In this model, the upper branch is the standard three-parameter solid model and the lower branch is a modified Maxwell model which contains a strain-stiffening spring element and a dashpot element connected in series. The new dashpot element $c_{1}$ is to introduce variable transition to strain-stiffening behaviour for the MRE base isolator. The mathematical model is given by:

$$
\begin{gathered}
F=k_{1} y+\alpha z^{3} \\
k_{1} y=k_{0}(x-y)+c_{0}(\dot{x}-\dot{y}) \\
\alpha z^{3}=c_{1}(\dot{x}-\dot{z})
\end{gathered}
$$

Rearranging equation 12 and 13 obtains

$$
\dot{z}=-\frac{\alpha}{c_{1}} z^{3}+\dot{x}
$$

Where, $c_{0}, k_{0}, c_{1}, k_{1}$ and $\alpha$ are the model parameter to be identified. 


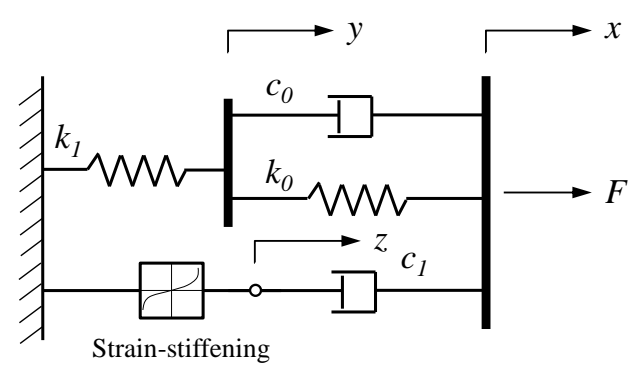

Figure 11 Modified strain-stiffening mechanical model

The modified strain-stiffening model described by equations 14-16 was also fit to the same experimental data used in previous models. Figure 12 shows the comparison between the experimental data and the proposed model. Due to the introduction of additional dashpot $\mathrm{c}_{1}$, smooth transition from elastic spring to strain-stiffening spring is realized in the model. Comparison indicates that this model can well capture the behaviour of the MRE base isolator at all displacement.

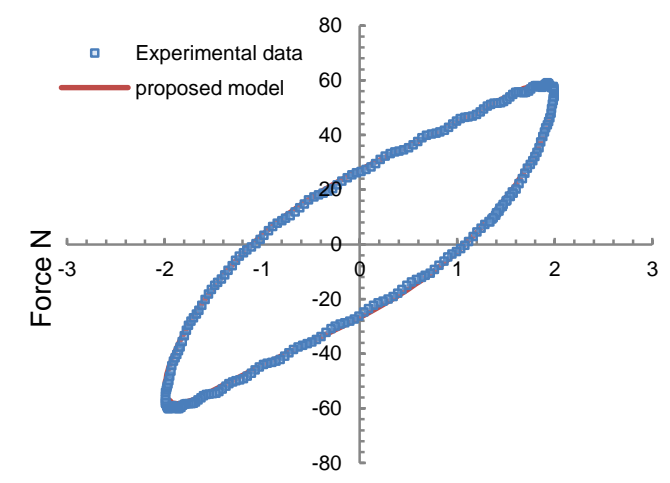

Displacement $\mathrm{mm}$

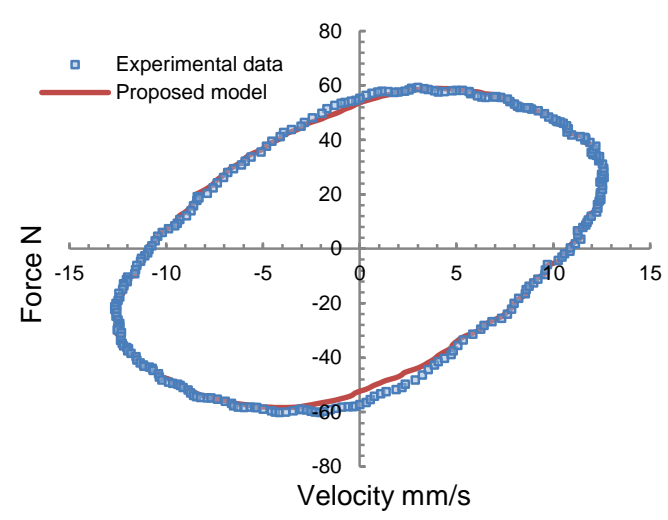

a) Small displacement

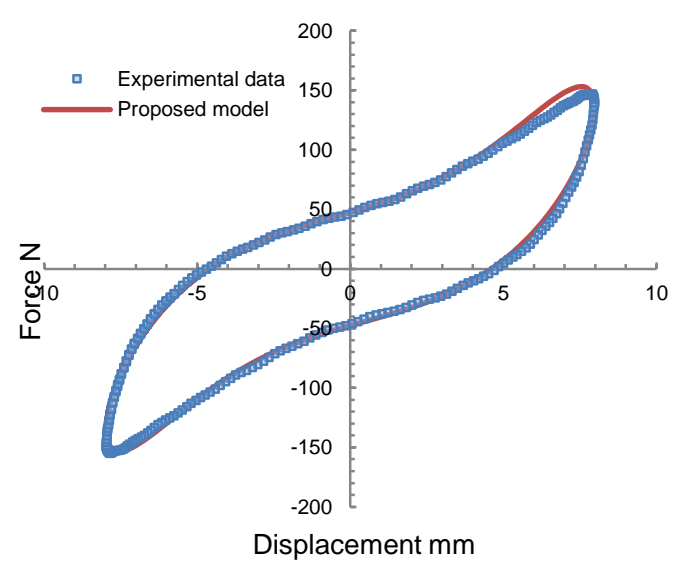

b) Large displacement

Figure 12. Comparison between the experimental data and model prediction for modified strain-stiffening model 2

\section{Modeling the adaptive MRE base isolator with input currents}

Upon the identification of a fidelity model for MRE base isolator, the next step is to extend the mechanical model to phenomenological model by introducing field-dependent modeling. To achieve this, a parametric study on the proposed model is conducted firstly. For MRE base isolator, magnetic field variation due to its lateral deformation should also be considered. Therefore, finite element 
analysis is to undertake to investigate the influence of lateral deformation. Finally, field-dependent parameters are introduced into the model to complete the modeling process.

\subsection{Model parametric analysis}

In this section, parametric study of the proposed strain-stiffening model is explored. The focus of this section is to investigate the infulence of five model parameters on the shapes and sizes of the hysteresis loop. Figure 13 shows the force-displacement and force-velocity relationships of the strain-stiffening element $F_{s t}=\alpha x^{3}$. The strain-stiffening element is a nonlinear elastic spring element. When $|x|<1$, the slope of the force-displacement curve increases slightly with $x$. In the range of $|x|>1$, the force of the strain-stiffening element increase dramatically. This model has a fixed turning point of $|x|=1$, which explains the inefficiency of the model proposed by equations 11 and 12 for predicting the performance of MRE base isolator under different lateral displacement. The force-velocity loop of the strain-stiffening element has two sharp ends at maximum positive and negative velocities, where the displacement is close to zero. The model parameters $\alpha, c_{1}$ and $c_{0}$ modify the turning point and sharpe ends in the hysteresis loop.
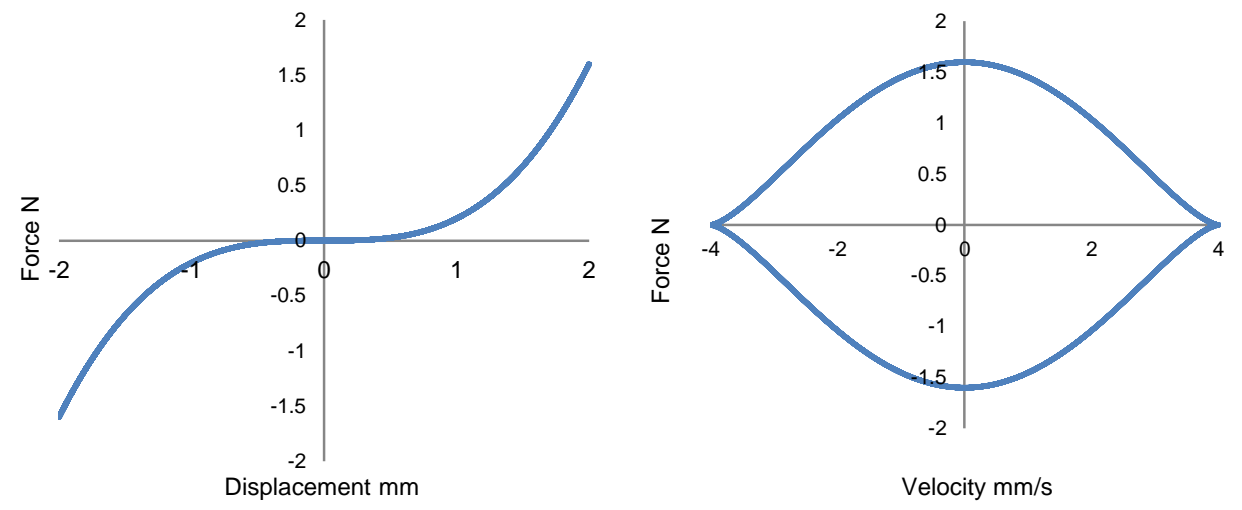

Figure 13. Characteresitics of the strain-stiffening element

To investigate the contribution of each model parameter on the hysteresis loop, a base model was chosen when $\alpha=0.5, c_{0}=2, k_{0}=0.2, c_{1}=12$ and $k_{1}=7$. The hysteresis loop denoted by the proposed model, i.e. equations 14-16, is determined by the upper branch, i.e. standard three parameter solid model (equation 12), and the lower branch, i.e. the in-series connected strainstiffening element and a dashpot (equation 13). The upper branch of the model determines the basic shape of the viscoelastic loop while the lower branch modifies the loop by creating the strainstiffening behavior (by controlling $\alpha$ ) with changable turning point (by controlling $\alpha$ and $c_{1}$ ). In the following section, the authors investigate the parameters in the lower branch, $\alpha$ and $c_{1}$, and parameters in the upper branch, $c_{0}, k_{0}$ and $k_{1}$, respectively.

Figure 14 shows the three sets of force data and hysteresis loops corresponding to three $\alpha$ values, i.e. $0,0.5$ and 1.5 , respectively. When $\alpha=0$, the model does not possess strain-stiffening behavior and represents typical viscoelastic behavior. When $\alpha$ increases, the strain-stiffening in the hysteresis loop becomes more obvious in force-displacement loop, which not only changes the slope of the hysteresis loop at large displacement but also the maximum force. In the force-velocity loops, they coincide in the left and right ends of the loops which stand for maximum positive and negative velocities. At these locations, the displacement is close to zero while the absolute velocity is maximum. Here, the strain-stiffening behavior disappears while the damping force dominants. Increase of $\alpha$ also enlarges the enalosed area of the force-velocity loop. Figure 15 shows three hysteresis curves of the MRE base isolator for three sets of $c_{1}$ values, i.e. 0.1, 1.0 and 100, respectively. Here, the model parameter $\alpha=3$. Equations 13 and 14 indicate that parameter $\alpha$ and $c_{1}$ 
determine the shape of $\mathrm{z}$ which accordingly affects the strain-stiffening behavior of the model. In force-velocity loops, it shows that increase on $c_{1}$ also enlarges the enclosed area.
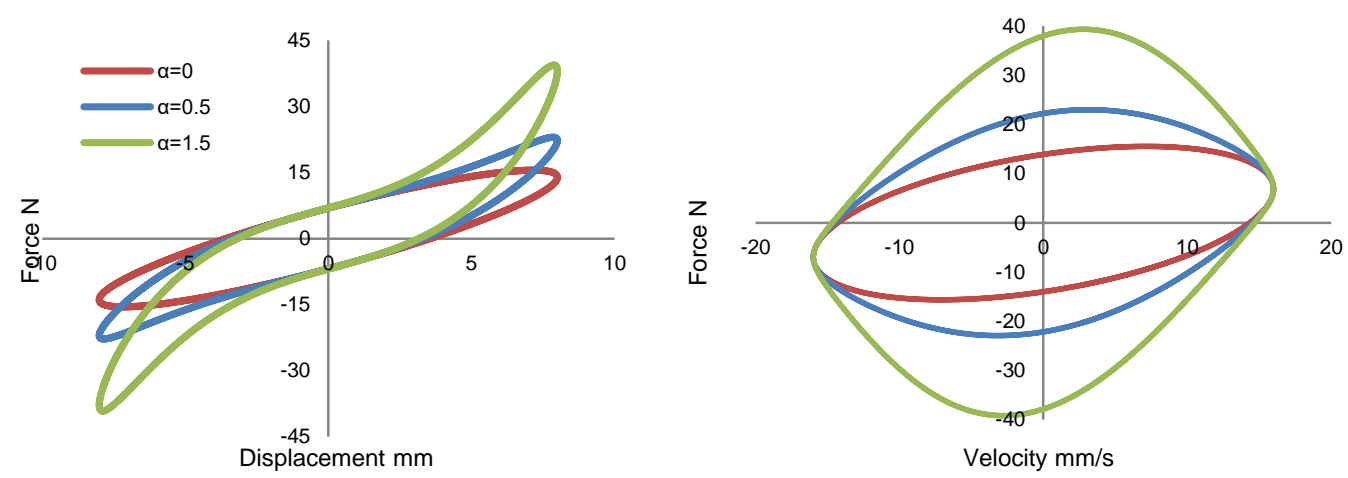

Figure 14 parameter $\alpha$ and the hysteresis loop
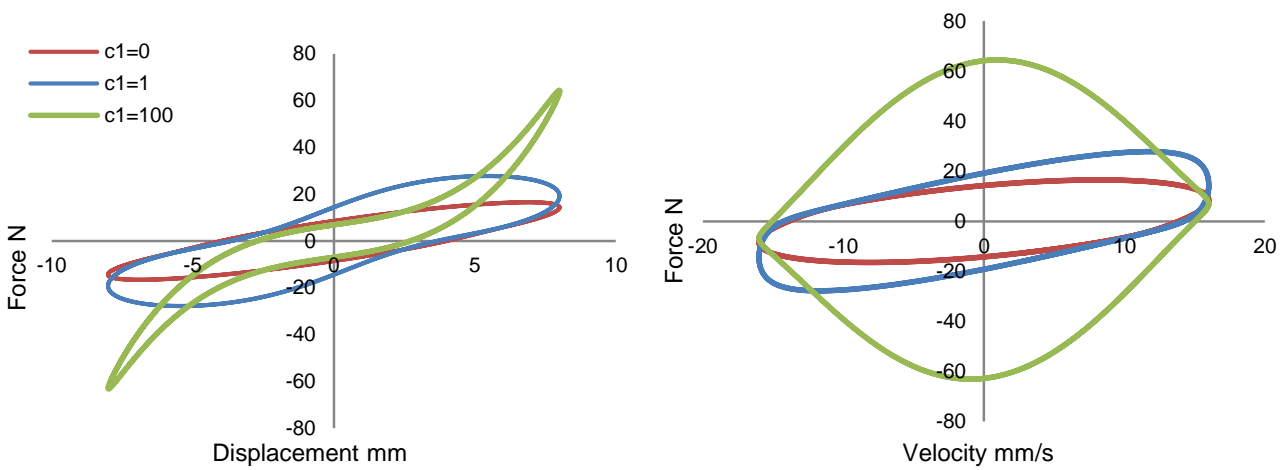

Figure 15 Parameter $c_{1}$ and the hysteresis loop

Three parameters in the upper section of the model, $c_{0}, k_{0}$ and $k_{1}$, affect the sizes and shapes of the linear viscoelastic loop of the three-parameter solid model upon which the modified model is built. Figures 16-18 are the hystersis loops of the proposed model for different sets of model parameters, $c_{0}, k_{0}$ and $k_{1}$, indicated in the figures, respectively. Equations 10 and 12 are the mathematical expression of the upper branch of the model. It should be noted that in the three-parameter model alone, its shape is decided by the values of $-\left(k_{0}+k_{1}\right) / c_{0}$ and $k_{0} / c_{0}$. While, the characteristics of the hysteresis loop are decided by both upper and lower branches.

As observed in figure 16, increase of $c_{0}$ leads to increase of the effective stiffness, i.e. slope of the loop, and changes the energy dissipation of the model. More importantly, change of $c_{0}$ modifies the sharpness of two ends of the force-velocity loop. Increase of $\mathrm{k}_{0}$ and $\mathrm{k}_{1}$ leads to the increase of the peak force in force-displacement loop thus creates increase of effective stiffness, shown in figures 17 and 18. However, their impacts on the shapes of force-velocity loops are quite different, as donated by the black arrows in figures 17 and 18. Changes of values of $k_{1}$ also significantly modify the enclosed area (energy dissipation) of force-displacement loop.
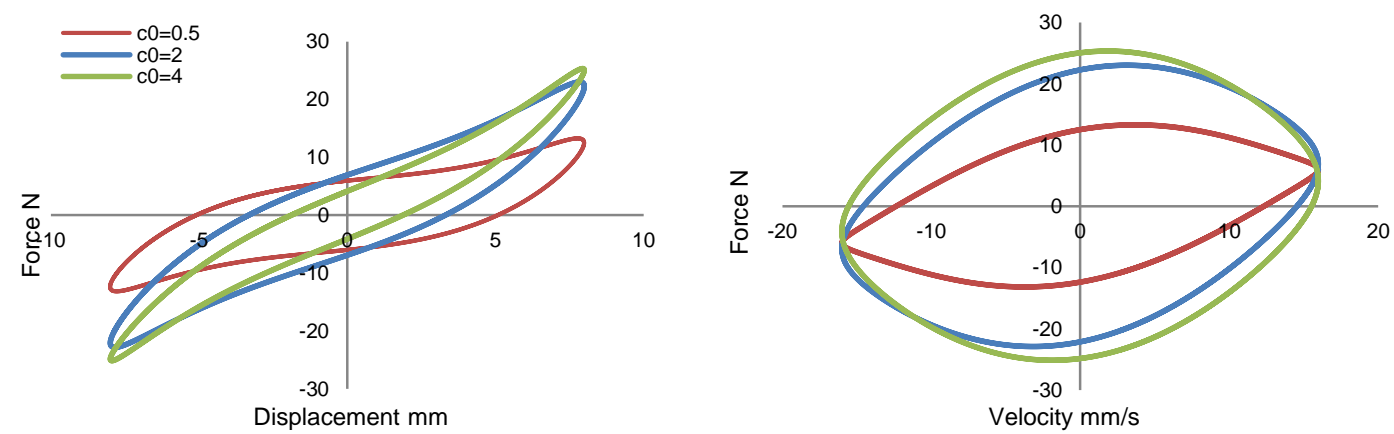
Figure 16 Parameter $c_{0}$ and the hysteresis loop
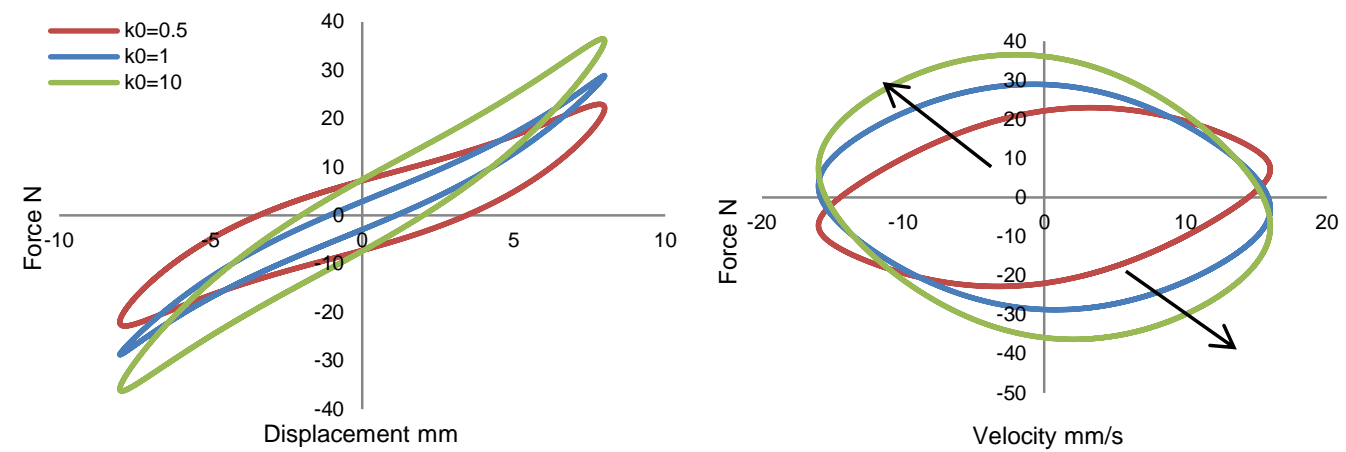

Figure 17 Parameter $k_{0}$ and the hysteresis loop
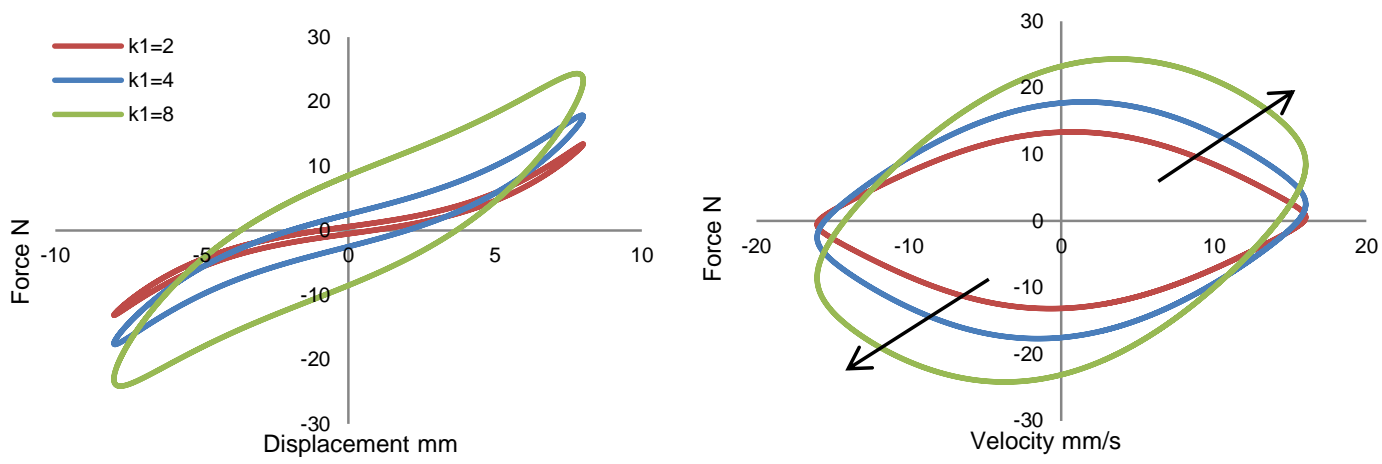

Figure 18 Parameter $k_{1}$ and the hysteresis loop

\subsection{Effect of lateral deformation on magnetic field}

In the MRE base isolator, MRE laminated structure (including multi-layer MRE and steel layers) serves as the core of the magnetic circuit. During the dynamic testing, the MRE laminated structure deforms along with the external motion, as shown in Figure 19. Figure 19 also shows the sketch of magnetic field distribution inside the MRE seismic isolator. The rectangular shape in figure 19a represents original position of the MRE seismic isolator when no deformation occurs. The parallelogram shape in figure 19b, including a rectangular area and two dashed triangular areas, represents the shape of MRE seismic isolator after certain deformation. It is expected that the magnetic field inside the MRE materials changes because of the motion of the device. This is clearly different from the magnetic field inside the MR damper in which the magnetic field should maintain constant during the motion the MR damper. The characteristics of the magnetic field due to the device motion should be considered in the modeling of the MRE seismic isolator performance later on.
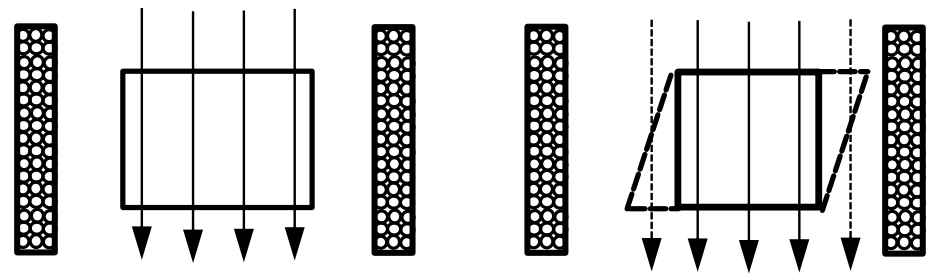

Figure 19 Magnetic field distributions in the seismic isolator (a. original position of the MRE seismic isolator. b. MRE seismic isolator deform with certain amplitude)

To evaluate magnetic field distribution in different layers, finite element analysis is conducted utilizing ANSYS Maxwell software[50]. A 3-dimensional finite element model is built. This device contains 25 laminated MRE layers. In the model, the MRE layers are named from top to bottom, i.e. 
the top layer is No.1 and the bottom layer is No. 25. Magnetic property of the MRE used in the simulation can be found in [51]. Figure 20 is the magnetic field distribution in the MRE base isolator for four deformations, i.e. $0 \mathrm{~mm}, 5 \mathrm{~mm}, 10 \mathrm{~mm}$ and $15 \mathrm{~mm}$, respectively. The results show that the magnetic field inside the MRE layers is uniform and deformation of the device does affect the magnetic field. To quantify the magnetic field under four deformations, average magnetic field intensity (B field) in five MRE layers, i.e. No. 1, 7, 13, 19 and 25, is calculated. The magnetic field data is plotted against deformation in Figure 21.

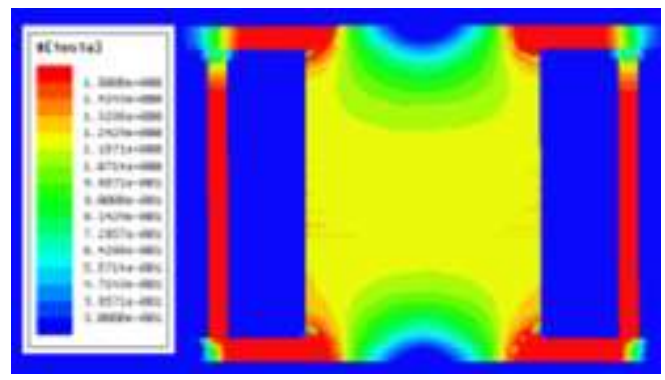

(i) deformation $=0 \mathrm{~mm}$

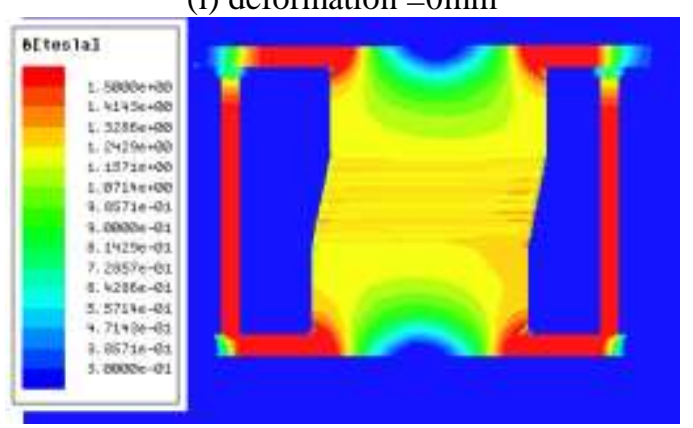

(iii) deformation $=0 \mathrm{~mm}$

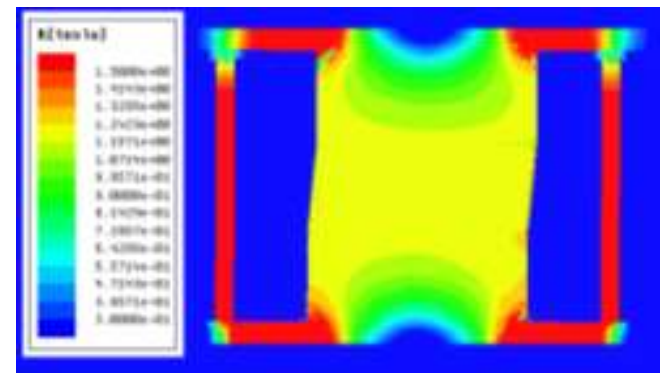

(ii) deformation $=5 \mathrm{~mm}$

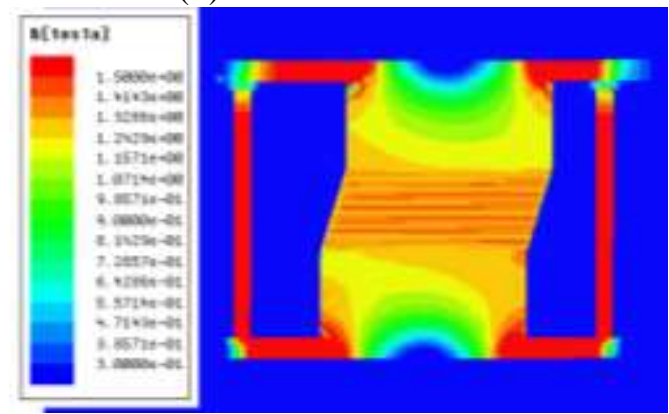

(iv) deformation $=15 \mathrm{~mm}$

Figure 20 Finite element analysis results $(\mathrm{I}=2 \mathrm{~A})$ for different deformation

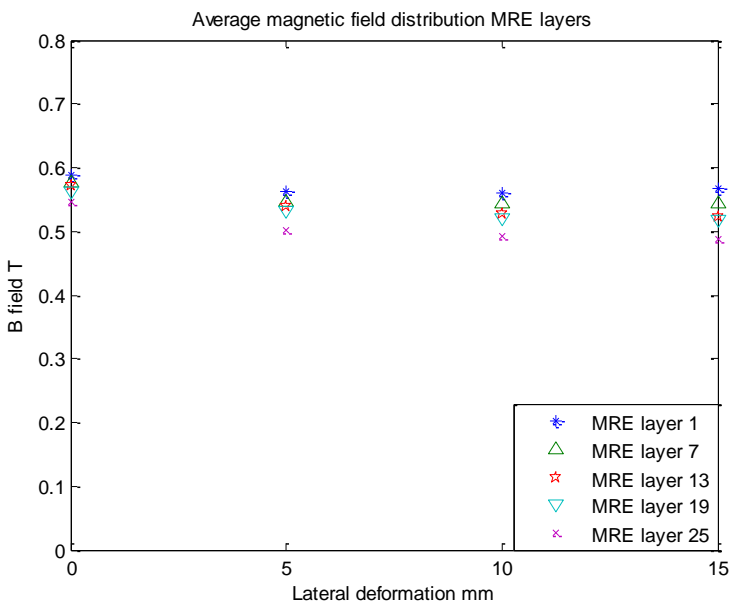

Figure 21 Magnetic field distributions in MRE layers at different lateral deformations (I=1A)

The results show that the magnetic field intensity decreases from top MRE layer to underneath layer. While deformation increases, the magnetic field intensity also slight decreases. For example, the B field in MRE layer 1 drops from $0.588 \mathrm{~T}$ for no deformation to $0.568 \mathrm{~T}$ at $15 \mathrm{~mm}$ deformation. However, since in the testing, only $8 \mathrm{~mm}$ deformation occurs. The B field drop is less than 0.01T which is a neglectable percentage of $1.7 \%$. Hence, effect of lateral deformation on the magnetic field distribution for modeling of MRE base isolator in this paper is not considered. However, if the deformation of the MRE base isolator leads to considerable decrease on the magnetic field, a linear assumption can be used in the modeling. The model parameters are subjected to a linear factor $\mu$ [52]: 


$$
\mu=1-\epsilon x
$$

Where, $x$ is the real-time displacement of the MRE base isolator, $\epsilon$ is the scale parameter to be determined by the finite element data.

\subsection{Field-dependent modeling}

In the previous sections, the effectiveness of the strain-stiffening model has been validated using sinusoidal loadings. In the real applications, the excitations are rather random or unexpected. Therefore, the proposed model needs to be further evaluated under random displacement inputs. To this purpose, shake table testing with two random displacement inputs have been conducted. These inputs include: (1) a 60s random input with wide-spread frequency range from $0-20 \mathrm{~Hz}$, as shown in figure 22 (only display 0-5s); (2) El-Centro earthquake input with duration of 60s, figure 23. During each testing, the applied currents were holding constant values, as $0 \mathrm{~A}, 1 \mathrm{~A}, 2 \mathrm{~A}$ and $3 \mathrm{~A}$.

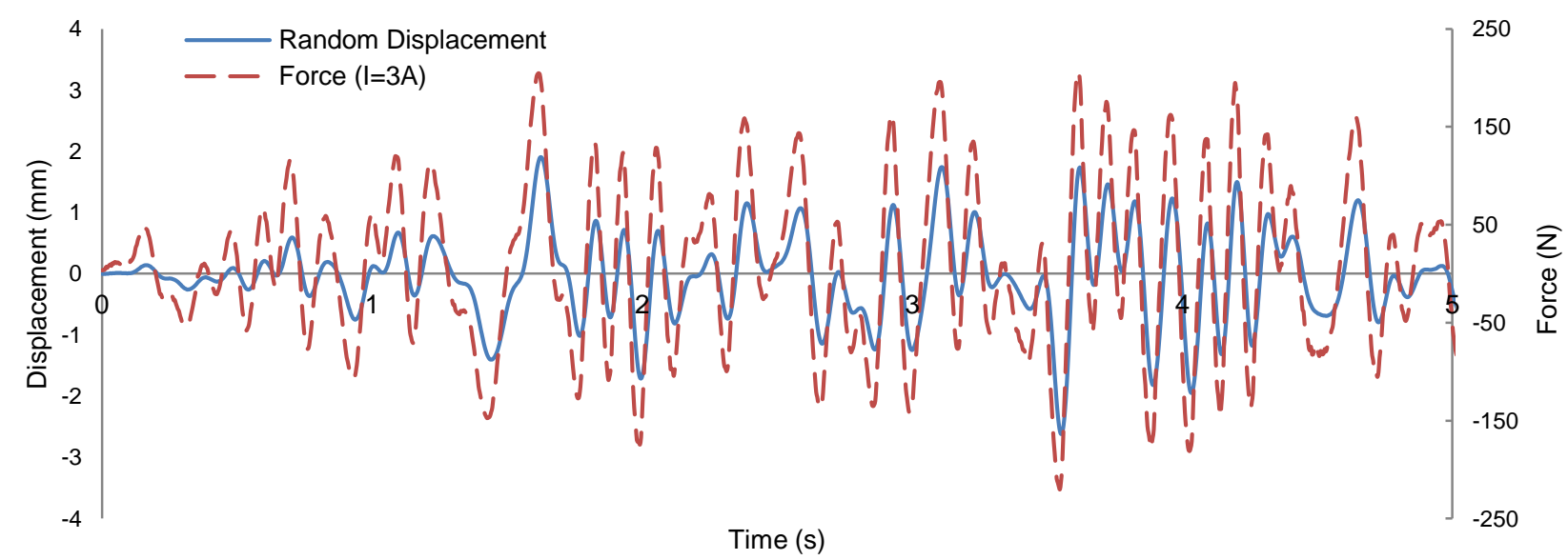

Figure 22. Random displacement input and force generated from the base isolator

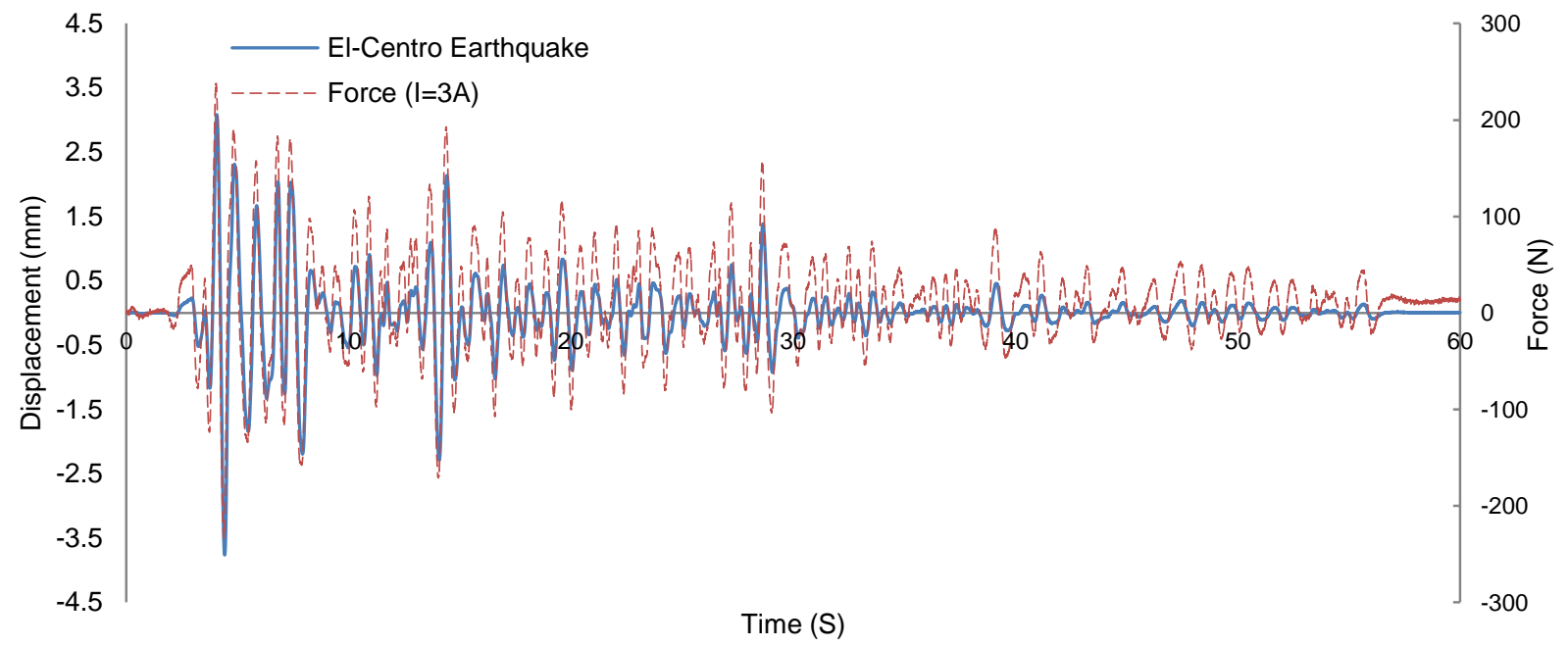

Figure 23. El-Centro earthquake input and force generated from the base isolator

In order to obtain optimized parameters for the proposed model under each set of input, objective function for the optimization is set as: 


$$
E_{\text {err }}=\int_{0}^{T}\left|F_{\text {exp }}^{2}-F_{\text {mod }}^{2}\right|
$$

Where, $F_{\text {exp }}$ is the force from MRE base isolator by experimental testing and $F_{\text {mod }}$ is the force prediction by the proposed model. In the numerical optimization, a Generalized-Reduced-Gradient (GRG) nonlinear method [53] is used to find the best fit to minimize the objective function $E_{\text {err }}$. Upon the identification of model parameters for each input, a set of optimal model parameters were chosen to better capture both random displacement input and EI-Centro earthquake input.

To take account of the influence of the magnetic field, following model with field-dependent variable, i.e. current I, is proposed:

$$
\begin{gathered}
c_{0}=c_{0 a} I+c_{0 b} \\
k_{0}=k_{0 a} I+k_{0 b} \\
c_{1}=c_{1 a} I+c_{1 b} \\
k_{1}=k_{1 a} I+k_{1 b} \\
\alpha=\alpha_{a} I+\alpha_{b}
\end{gathered}
$$

The model parameters for Equations 17-21 are identified as shown in Table 2. Figures 24 and 25 deliver the comparison between the experimental data and proposed model using the optimal model parameters for both random input and earthquake input. To further evaluate the model accuracy, following index is used to compare the model prediction with experimental data [54]:

$$
C C=\frac{\sum_{i=1}^{N}\left[F_{a}(i)-\overline{F_{a}}\right] \cdot\left[F_{p}(i)-\overline{F_{p}}\right]}{\sqrt{\sum_{i=1}^{N}\left[F_{a}(i)-\overline{F_{a}}\right]^{2}} \cdot \sqrt{\sum_{i=1}^{N}\left[F_{p}(i)-\overline{F_{p}}\right]^{2}}}
$$

where $F_{a}(k)$ and $F_{p}(k)$ denote the experimental data and predictions from the proposed model,

\begin{tabular}{|c|c|c|}
\hline Parameters & value & Unit \\
\hline$c_{O a}$ & -0.9687 & $N . s /(m m I)$ \\
\hline$c_{O b}$ & 14.521 & N.s/mm \\
\hline$k_{0 a}$ & 38.565 & $N /(m m I)$ \\
\hline$k_{o b}$ & 22.108 & $\mathrm{~N} / \mathrm{mm}$ \\
\hline$c_{1 a}$ & 0.0489 & $N . s /(m m I)$ \\
\hline$c_{1 b}$ & 1.4895 & N.s/mm \\
\hline$k_{l a}$ & 55.439 & $N /(m m I)$ \\
\hline$k_{1 b}$ & 35.927 & $\mathrm{~N} / \mathrm{mm}$ \\
\hline$\alpha_{a}$ & -0.0256 & $N /\left(\mathrm{mm}^{3} \mathrm{I}\right)$ \\
\hline$\alpha_{b}$ & 0.1332 & $\mathrm{~N} / \mathrm{mm}^{3}$ \\
\hline
\end{tabular}
respectively; $\bar{F}_{a}$ and $\bar{F}_{p}$ denote the average values of measured and predicted responses, respectively; $N$ denotes the total number of measured data. Generally, the higher the value of $C C$ is, the better the agreement between two responses will be. For both random and El-Centro inputs, the proposed model achieved the CC indies of 0.9623 and 0.9589 , respectively. It shows that the proposed model can well describe the behavior of the MRE base isolator under various inputs.

Table 2. Model parameters for generalized model 


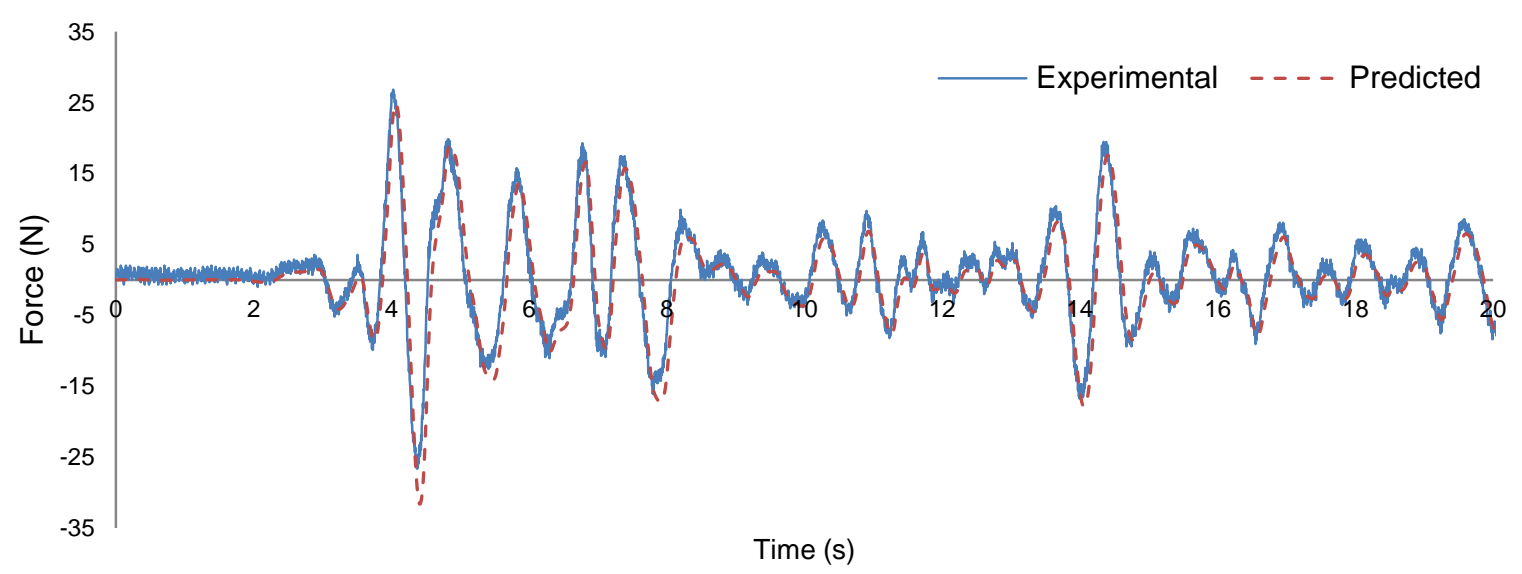

Figure 24. Comparison between the experimental data and generalized model under El-Centro earthquake input (I=0A)

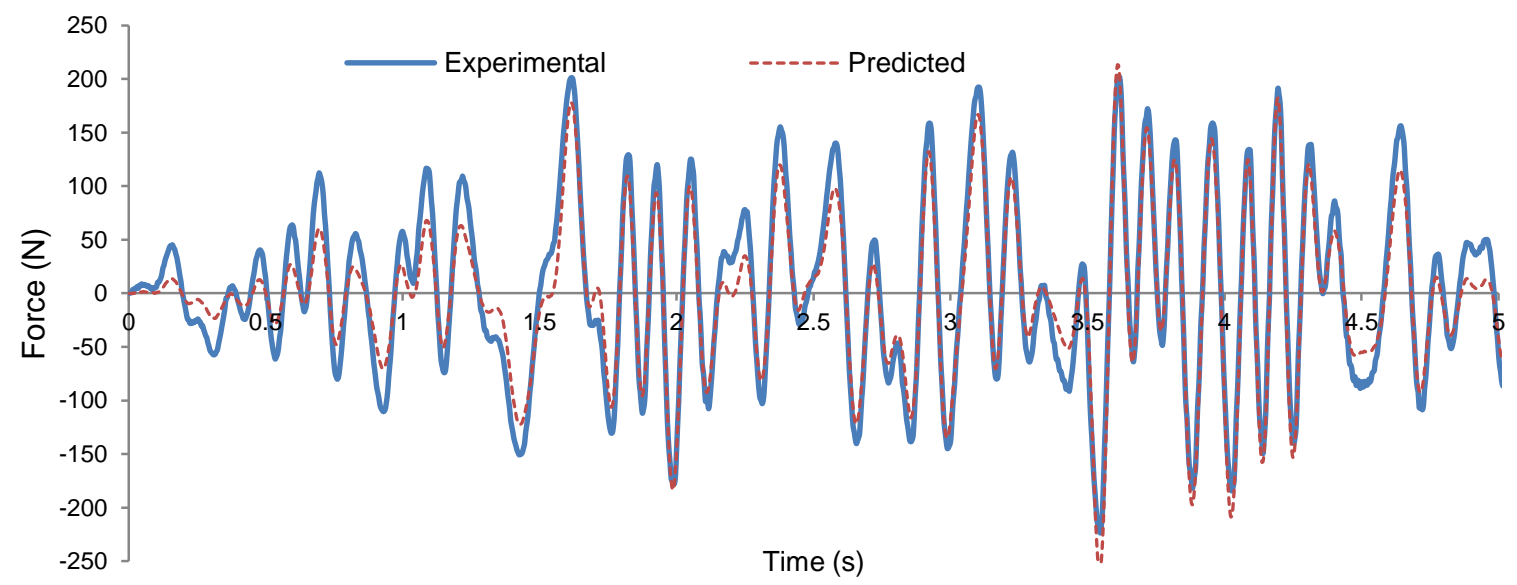

(a) Force data- time history

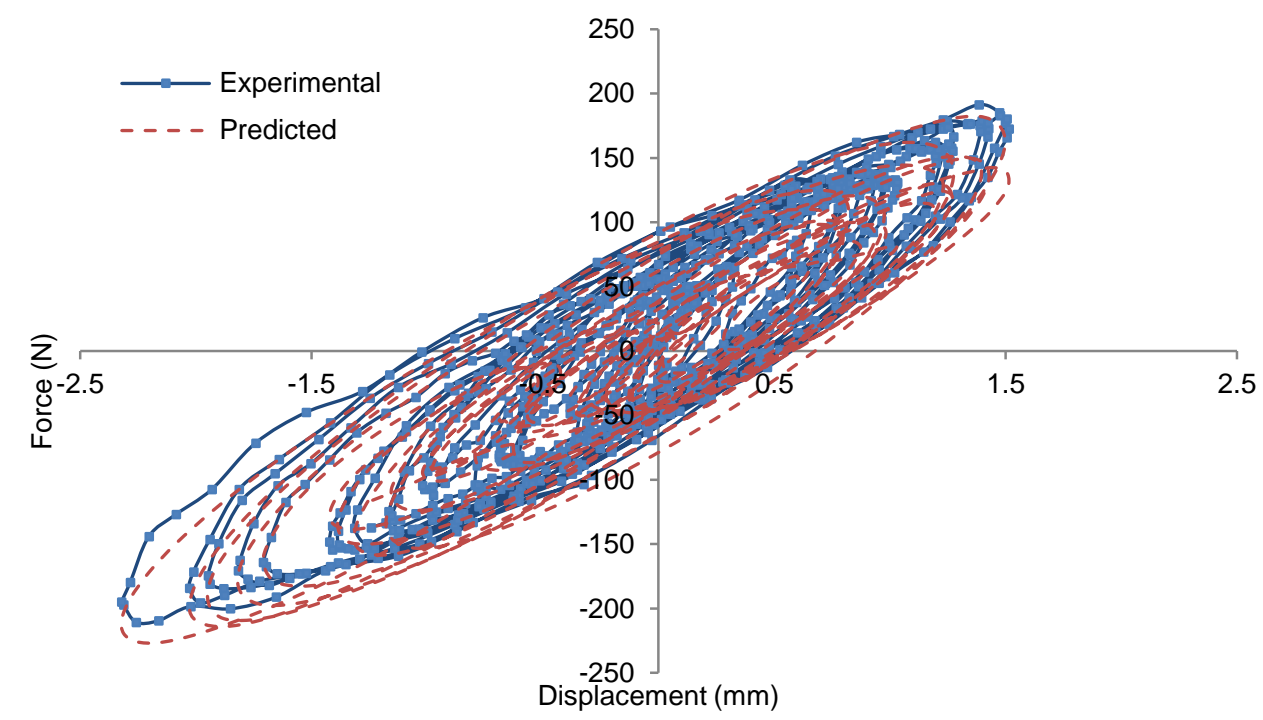

(b) Force vs displacement

Figure 25. Comparison between the experimental data and generalized model under random input (I=2A)

\section{Conclusions}

A quest on a new generation of intelligent base isolation systems capable of adapting unknown types of earthquakes to provide reliable protection to structures and their contents has been continued for 
decades. To effectively decouple various unknown destructive motions from civil structures to be protected, ability to change stiffness and damping of base isolators in real-time is essential for the development of the new generation of the base isolation system. To achieve this, researchers have turned attentions to the development of new base isolators capable of varying their stiffness instantly, including a new adaptive base isolator utilizing the advantages of field-dependent properties of MR elastomer. These new MRE isolators have demonstrated many advantages include high adjustable range, fast response, low power requirement and stability over a broad temperature range. With these attractive features, MRE base isolators show a great potential to be accepted and implemented in structural control of the new generation of base isolation system. To take advantage of unique features of MRE base isolator for designing an adaptive base isolation system, an accurate and reliable MRE base isolator model is required.

Following successful development of an adaptive MRE base isolator and subsequence dynamic testing to characterize behavior of the isolator by authors, this paper made an effort to capture unique behaviors of the MRE base isolator by modeling. Classical viscoelastic model was fit to the experimental data and results showed its inadequateness for predicting the strain-stiffening behavior. To portray the unique behavior existing in MRE base isolator, a new strain-stiffening element was proposed after a review of mechanical models of traditional laminated rubber bearing. A novel ratedependent mechanical model was proposed by incorporating the strain-stiffening element. Detailed model parametric study was conducted to investigate the influence of each model parameter to the size and shapes of the hysteresis loop. Finite element analysis was also undertaken to investigate the effect of lateral deformation on the magnetic field inside the MRE materials. Finally, field-dependent modeling procedure was introduced into the proposed mechanical model to correlate the experimental data with applied currents. Evaluation and comparison show that the proposed model is able to accurately predict the unique behavior of the MRE base isolator for various displacements and applied current. Therefore, it is feasible to adapt this model for the control design and analysis of an intelligent base isolation system incorporated with the adaptive MRE isolators.

\section{Acknowledgement}

This work was supported by Australian Research Council through Discovery Project (Grant No. DP150102636).

\section{References:}

[1]. Naeim F and J M. Kelly 1999 Design of seismic isolated structures: from theory to practice (Hoboken, NJ: John Wiley and Sons)

[2]. Komodromos P 2001 Seismic isolation for earthquake resistant structures (Southampton, UK: WIT Press)

[3]. Kelly J M 1990 Base isolation: linear theory and design Earthq. Spectra 6 223-244.

[4]. Kelly J M 2002 Seismic isolation systems for developing countries Earthq. Spectra 18 385-406.

[5]. Pan P, Zamfirescu D, Nakashima M, Nakayasu N and Kashiwa H 2005 Base-isolation design practice in Japan: introduction to the post-Kobe approach J. Earthq. Eng. 9 147-71.

[6]. Spencer B F, Dyke S J, Sain M K and Carlson J D 1997 Phenomenological model for magnetorheological dampers J. Eng. Mech. 123 230-8

[7]. Ramallo J C, Johnson E A and Spencer B F 2002 "Smart" base isolation systems J. Eng. Mech. 128 1088-99.

[8]. Spencer B F and Nagarajaiah S 2003 State of the art of structural control J. Struc. Eng. 129 845-56.

[9]. Kelly J M 1999 The role of damping in seismic isolation Earthq. Eng. Struct. Dyn. 28 3-20

[10]. Nagarajaiah S and Sahasrabudhe S 2006 Seismic response control of smart sliding isolated buildings using variable stiffness systems: experimental and numerical study Earthq. Eng. Struct. Dyn. 35 177-97.

[11]. Sahasrabudhe S and Nagarajaiah S 2005 Effectiveness of variable stiffness systems in base-isolated bridges subjected to near-fault earthquakes: an experimental and analytical study J. Intell. Mater. Syst. Struct 16 743-56.

[12]. Narasimhan S and Nagarajaiah S 2005 STFT algorithm for semiactive control of base isolated buildings with variable stiffness isolation systems subjected to near fault earthquakes Eng. Struct. 27 514-23. 
[13]. Lavallee P M 2008 Applications of force actuators and variable stiffness systems with base isolation. Massachusetts Institute of Technology: Cambridge, MA.

[14]. Pasala D, Sarlis A, Nagarajaiah S, Reinhorn A, Constantinou M and Taylor D 2013 Adaptive negative stiffness: new structural modification approach for seismic protection J. Struc. Eng. 139 1112-23.

[15]. Li Y, Li J, Li W and Du H 2014 A state-of-the-art review on magnetorheological elastomer devices Smart Mater. Struct. 23123001.

[16]. Behrooz M, Wang X and Gordaninejad F 2014 Performance of a new magnetorheological elastomer isolation system Smart Mater. Struct. 23045014

[17]. Liao G J, Gong X L and Xuan S H 2014 Phase based stiffness tuning algorithm for a magnetorheological elastomer dynamic vibration absorber Smart Mater. Struct. 23015016

[18]. Liao G J, Gong X L, Xuan S H, Kang C J and Zong L H 2011 Development of a real-time tunable stiffness and damping vibration isolator based on magnetorheological elastomer J. Intell. Mater. Syst. Struct. 23 25-33

[19]. Liao G J, Gong X L, Kang C J and Xuan S H 2011 The design of an active-adaptive tuned vibration absorber based on magnetorheological elastomer and its vibration attenuation performance Smart Mater. Struct. 20 075015

[20]. Li Y, Li J, Li W and Samali B 2013 Development and characterization of a magnetorheological elastomer based adaptive seismic isolator Smart Mater. Struct. 22035005.

[21]. Li Y, Li J, Tian T and Li W 2013 A highly adjustable magnetorheological elastomer base isolator for applications of real-time adaptive control Smart Mater. Struct. 22095020.

[22]. Li WH, Zhou Y and Tian T 2010 Viscoelastic properties of MR elastomers under harmonic loading Rheol. Acta 49 733-40.

[23]. Popp K, Kroger M, Li W, Zhang X and Kosasih P 2010 MRE properties under shear and squeeze modes and applications J. Intell. Mater. Syst. Struct 21 1471-7.

[24]. Koo J H, Dawson A and Jung H J 2012 Characterization of actuation properties of magneto-rheological elastomers with embedded hard magnetic particles J. Intell. Mater. Syst. Struct 231049-54.

[25]. Gong X L, Zhang X Z and Zhang P Q 2005 Fabrication and characterization of isotropic magnetorheological elastomers Polym. Test. 24 669-76.

[26]. AASHTO 1999 Guide specifications for seismic isolation design. Washington D. C. American Association of State Highway and Transportation Officials. (AASHTO)

[27]. Summary of evaluation findings for the testing of seismic isolation and energy dissipating devices. Reston, VA: American Society of Civil Engineers c1999; 20-50.

[28]. Sjoberg M and Kari L 2002 Non-linear behaviour of a rubber isolator system using fractional derivatives Vehicle Syst. Dyn. 37 217-36.

[29]. Iizuka M 2000 A macroscopic model for predicting large-deformation behaviors of laminated rubber bearings Eng. Struct. 22 323-34.

[30]. Ramberg W and Osgood W R 1943 Description of stress-strain curves by three parameters. Technical Note No. 902, National Advisory Committee for Aeronautics, Washington DC.

[31]. Yu Y, Li Y and Li J 2015 Parameter identification and sensitivity analysis of an improved LuGre friction model for magnetorheological elastomer base isolator Meccanica 50 2691-707

[32]. Lee K S, Sause R, Ricles J, AB-Malek K and Lu L W 2004 Non-linear rate-dependent hysteresis model for structural dampers made from ultra-high damping natural rubber J. Rubber Res. 7 79-103.

[33]. Yoshida J, Abe M and Fujino Y 2004 Constitutive model of high-damping rubber materials J. Eng. Mech. 130 129-41.

[34]. Kikuchi M and Aiken I D 1997 An analytical hysteresis model for elastomeric seismic isolation bearings Earthq. Eng. Struct. Dyn. 26 215-31.

[35]. Tsai, C S, Chiang T C, Chen B J and Lin S B 2003 An advanced analytical model for high-damping rubber bearings Earthq. Eng. Struct. Dyn. 32 1373-87.

[36]. Abe M, Yoshida J and Fujino Y 2004 Multiaxial behaviors of laminated rubber bearings and their modelling. I: experimental study J. Eng. Mech. 130 1119-32.

[37]. Abe M, Yoshida J and Fujino Y 2004 Multiaxial behaviors of laminated rubber bearings and their modelling. II: modeling J. Eng. Mech. 130 1133-44. 
[38]. Sause R, Lee K S, Ricles J, AB-Malek K and Lu L W 2001 Non-linear rate-dependent hysteresis model for ultra-high damping NR structural dampers J. Rubber Res. 4 222-44.

[39]. Bhuiyan AR, Okui Y, Mitamura H and Imai T 2009 A rheology model of high damping rubber bearings for seismic analysis: identification of nonlinear viscosity Int. J. Solids Struct 46 1778-92.

[40]. Hwang J S and Ku S W 1997 Analytical modeling of high damping rubber bearings J. Struct. Eng. 123 102936.

[41]. Yamamoto S, Kikuchi M, Ueda M and Aiken I D 2009 A mechanical model for elastomeric seismic isolation bearings including the influence of axial load Earthq. Eng. Struct. Dyn. 38 157-80.

[42]. Yamamoto M, Minewaki S, Yoneda H and Higashino M 2012 Nonlinear behaviour of high-damping rubber bearings under horizontal bidirectional loading: full-scale tests and analytical modeling Earthq. Eng. Struct. Dyn. 41 1845-60.

[43]. Hwang J S, Wu J D, Pan T C and Yang G 2002 A mathematical hysteretic model for elastomeric isolation bearings Earthq. Eng. Struct. Dyn. 31 771-89.

[44]. Dall'Asta A and Ragni L 2008 Dynamic systems with high damping rubber: nonlinear behaviour and linear approximation Earthq. Eng. Struct. Dyn. 37 1511-26.

[45]. Pasquale G D and Sano T 1995 A constitutive model for high damping rubber bearings J Press. Vess. Technol. 117 53-8.

[46]. Jankowski R 2003 Nonlinear rate dependent model of high damping rubber bearing Bulletin Earthq. Eng. $1397-$ 403.

[47]. Dall'Asta A and Ragni L 2006 Experimental tests and analytical model of high damping rubber dissipating devices Eng. Struct. 28 1874-84.

[48]. Koh C and Kelly J M 1988 A simple mechanical model for elastomeric bearings used in base isolation Int. J. Mech. Sci. 30 933-44.

[49]. Ozdemir H 1976 Nonlinear transient dynamic analysis of yielding structures. Ph. D Dissertation, University of California, Berkeley.

[50]. $\quad \mathrm{Li} \mathrm{Y}$ and Li J 2015 Finite element design and analysis of adaptive base isolator utilizing laminated multiple magnetorheological elastomer layers J. Intell. Mater. Syst. Struct 26 1861-70

[51]. Zeng J, Guo Y, Li Y, Zhu J and Li J 2013 Two-dimensional magnetic property measurement for magnetorheological elastomer J. Appl. Phys. 113 17A919-17A919-3

[52]. Wang X and Gordaninejad F 2009 A new magnetorheological fluid-elastomer mount: phenomenological modelling and experimental study Smart Mater. Struct. 18095045.

[53]. Lasdon L S, Fox R L and Ratner M W 1973 Nonlinear Optimization Using the Generalized Reduced Gradient Method. Technical Memorandum No. 325: Case Western Reserve University.

[54]. Yu Y, Li Y, Li J and Gu X 2016 Self-adaptive step fruit fly algorithm optimized support vector regression model for dynamic response prediction of magnetorheological elastomer base isolator Neurocomputing 21141 52 